\title{
Dry eye after haematopoietic stem cell transplantation
}

Yoko Ogawa, Shinichiro Okamoto, Masatoshi Wakui, Reiko Watanabe, Masakazu Yamada, Mami Yoshino, Masafumi Ono, Hao-Yung Yang, Yukihiko Mashima, Yoshihisa Oguchi, Yasuo Ikeda, Kazuo Tsubota
Department of

Ophthalmology, Keio

University, School of

Medicine, Tokyo, Japan

Y Ogawa

$M$ Yamada

M Yoshino

M Ono

H-Y Yang

Y Mashima

Y Oguchi

Keio BMT Program,

Division of

Haematology,

Department of

Medicine, Keio

University, School of

Medicine, Tokyo, Japan

S Okamoto

M Wakui

$\mathrm{R}$ Watanabe

Y Ikeda

Department of

Ophthalmology, Tokyo

Dental College, Chiba,

Japan

K Tsubota

Correspondence to: Kazuo Tsubota, MD, Department of

Ophthalmology, Tokyo

Dental College, 5-11-13,

Sugano, Ichikawa City,

Chiba, 272-8513, Japan.

Accepted for publication 28 June 1999

\begin{abstract}
Aims-To determine the incidence, natural course, and severity of dry eye occurring or worsening after haematopoietic stem cell transplantation (SCT).

Methods-At a tertiary care hospital, 53 patients undergoing allogeneic or autologous SCT followed by at least 180 days of follow up were studied prospectively. Examination included grading of symptoms of dry eye, evaluation of ocular surface, tear break up time, and Schirmer tests with and without nasal stimulation. Meibomian gland secretion was also examined using a slit lamp while applying steady digital pressure.

Results-Of the 53 patients, 44 received allografts. Half of these patients (22) developed dry eye or their pre-existing dry eye worsened after SCT, while none of nine autograft recipients did. Onset of dry eye was 171 (SD 59) days after SCT. Two types of dry eye occurred. One $(n=10)$ was severe with ocular surface findings resembling Sjögren's syndrome and reduction of reflex tearing soon after onset. A mild type $(n=12)$ had unimpaired reflex tearing. Meibomian gland dysfunction (MGD) was more frequent and severe in patients with dry eye and chronic graft versus host disease (GVHD), and overall severity of dry eye was greater in patients with MGD and chronic GVHD.

Conclusions-Dry eye after SCT occurred only in allograft recipients, and was not evident in autograft recipients. The severe form of dry eye had a tendency to develop rapidly. Further study on the prediction and treatment of severe dry eye after SCT is necessary.

(Brf Ophthalmol 1999;83:1125-1130)
\end{abstract}

Haematopoietic stem cell transplantation (SCT) is an established treatment for many patients with life threatening haematological diseases. ${ }^{12}$ SCT has been increasingly used worldwide in the past two decades. Because many recipients of SCT become long term survivors, their quality of life and the possibility of late complications after SCT have become increasingly important. ${ }^{13}$ Dry eye has become recognised as a major symptom of chronic graft versus host disease (GVHD) with a significant impact on quality of life, although it is not life threatening. ${ }^{1-11}$ While several supportive measures have been developed and clinically applied, ${ }^{38}$ effective treatment and prophylaxis of dry eye has not yet been established.

In this prospective study, we sought to determine the incidence and natural course of dry eye and to identify risk factors for development of dry eye after SCT, with the ultimate goal of better prophylaxis and treatment of this ophthalmic complication.

\section{Patients and methods}

STUDY DESIGN

This study was part of a prospective survey evaluating the late effects of SCT. All patients underwent standardised clinical and ophthalmological evaluation as described below before SCT and 3, 6, 9, 12, 18, 24, and 30 months after transplantation, and on additional occasions as indicated. Between October 1995 and March 1998, 102 patients underwent SCT at Keio University Hospital. Of these patients, 53 (52.0\%) who had follow up periods of at least 180 days after SCT were included in this study. Five patients under 12 years old, 28 patients with shorter follow up periods, and 16 patients who died early in their course of transplant were excluded. Informed consent was obtained from all patients.

\section{CLINICAL EVALUATION}

Symptoms of dry eye, including ocular fatigue, dry eye sensation, a foreign body sensation, ocular pain, blurring, photophobia, red eye, discharge, heavy eye feeling, dull sensation, difficulty of eye opening, epiphora, an itchy sensation, a burning sensation, and dry mouth were graded by patients before and after SCT using a standard questionnaire. Each of the 15 symptoms were scored $0-5$ according to severity: 0 , none; 1 , occasional; 2 , constant, but mild and putting no restriction on activities of daily living (ADLs); 3, constant and moderate, but no restriction of ADLs; 4, constant and severe with some restriction of ADLs; 5, severe restriction of ADLs. ${ }^{12}$ The total maximum score was 75 points. Scores were compared before and after SCT.

The condition of the ocular surface was evaluated with a double staining method using $2 \mu \mathrm{l}$ of a mixed solution including preservative free $1 \%$ rose bengal and $1 \%$ fluorescein instilled by a micropipette..$^{13}$ The degree of rose bengal staining was quantified for the temporal and nasal conjunctiva and the cornea, on a scale of $0-3$ points. Fluorescein staining was also rated from 0 to 9 , but only for the cornea. ${ }^{14}$ Tear dynamics were assessed by five different methods ${ }^{15}$ including tear break up time (BUT), 
the Schirmer test, the cotton thread test, fluorescein clearance, and a Schirmer test with nasal stimulation. Three values for BUT were obtained at the time of double staining. The Schirmer test with nasal stimulation, which is considered to evoke maximal reflex tearing, ${ }^{16} 17$ was performed by applying a baby cotton swab to the nasal cavity. Meibomian gland secretions were examined using a slit lamp by application of a constant digital pressure. ${ }^{18}$ The lens and fundus were examined after pupillary dilatation with $1 \%$ tropicamide to look for other ophthalmic complications associated with SCT.

DIAGNOSTIC CRITERIA

Dry eye was diagnosed when patients had any sign of tear film instability (BUT $\leqslant 5$ seconds, Schirmer test $\leqslant 5 \mathrm{~mm}$, or cotton thread test $\leqslant 10 \mathrm{~mm}$ ), and any abnormality of the ocular surface (rose bengal score $\geqslant 3$, fluorescein score $\geqslant 1$ ), and/or symptoms of ocular irritation. ${ }^{19}$ We used the global diagnostic criteria of dry eye proposed by Lemp. ${ }^{20}$ Dry eye was diagnosed as a disorder of the tear film due to tear deficiency or excessive tear evaporation which caused damage to the interpalpebral ocular surface and was associated with or without symptoms of ocular discomfort. ${ }^{20}$ Blepharitis associated with infectious disease, blink disorders, disorders of the lid aperture and lid/globe congruity, other ocular surface disorders and contact lens induced tear film disorders were excluded from the diagnosis of dry eye in this study. Patients who had dry eye before SCT were considered to have sustained a dry eye incident positive only when the severity of dry eye worsened following transplantation. Severe dry eye was defined as reduced reflex tearing (Schirmer test with nasal stimulation $\leqslant 10 \mathrm{~mm}$ ) and ocular surface abnormality (rose bengal score $\geqslant 3$ and/or fluorescein score $\geqslant 1)$.

To assess obstruction of the meibomian gland orifice, digital pressure was applied on the lower tarsus, and expression of meibomian

Table 1 Patient characteristics by graft type and dry eye status

\begin{tabular}{llll}
\hline & & & \multicolumn{2}{l}{$\begin{array}{l}\text { Autograft } \\
\text { recipients }\end{array}$} \\
\cline { 2 - 3 } Dry eye after SCT & + & - & - \\
\cline { 2 - 3 } Number & 22 & 22 & 9 \\
Dry eye before SCT & 4 & 3 & 2 \\
Sex (F:M) & $9: 13$ & $7: 15$ & $8: 1$ \\
Age, mean (SD) & $36.0(8.0)$ & $27.0(10.7)$ & $42.9(8.8)$ \\
Stem cell source R:U & $12: 10$ & $17: 5$ & \\
Acute GVHD (\%) & $17(77.3 \%)$ & $11(50.0 \%)$ & 0 \\
Underlying diseases & & & \\
AML & 6 & 10 & 2 \\
ALL & 5 & 5 & 0 \\
CML & 5 & 4 & 0 \\
NHL & 0 & 0 & 4 \\
HD & 0 & 1 & 1 \\
MDS & 4 & 0 & 0 \\
MM & 2 & 0 & 1 \\
Aplastic anaemia & 0 & 2 & 0 \\
Breast Ca & 0 & 0 & 1 \\
& & &
\end{tabular}

SCT = stem cell transplantation; AML = acute myeloid leukaemia; $\mathrm{ALL}=$ acute lymphoblastic leukaemia; $\mathrm{CML}=$ chronic myeloid leukaemia; NHL = non-Hodgkin's lymphoma; HD = Hodgkin's disease; $\mathrm{MDS}=$ myelodysplastic syndrome; $M M=$ multiple myeloma; $\mathrm{Ca}=$ carcinoma; $\mathrm{R}=$ related donor; $\mathrm{U}=$ unrelated donor. secretion (meibum) was scored as follows: grade 0 , clear meibum easily expressed; grade 1 , cloudy meibum expressed with varying degrees of pressure; and grade 2, meibum not expressed even with heavy pressure. ${ }^{21}$

\section{STEM CELL TRANSPLANTATION}

The basic SCT protocol remained unchanged throughout the study period. Briefly, patients with a malignant disease were treated with four different preparative regimens. For recipients of allogeneic bone marrow from an HLA matched sibling, total body irradiation (TBI) and cytosine arabinoside (Ara-C) were used, except for the patients with chronic myelogenous leukaemia in chronic phase who received busulphan (BU) plus cyclophosphamide (CY). For recipients of allogeneic bone marrow from an HLA matched unrelated donor, TBI and CY were used with or without Ara-C regardless of primary disease. For recipients of autologous peripheral blood stem/progenitor cells, Ara-C, CY, etoposide, and ranimustine were used in most cases, except for patients with myeloma or breast carcinoma who received melphalan or BU plus thiotepa, respectively. For patients with severe aplastic anaemia, total lymphoid irradiation and CY were used. TBI (12 Gy) was applied by a linear accelerator, usually as six fractions over 3 consecutive days at a dose rate of $15 \mathrm{cGy} / \mathrm{min}$. No shielding was used over the eyes. Posttransplant graft versus host disease (GVHD) prophylaxis was attempted with either cyclosporine (CSP) and methotrexate (MTX) or tacrolimus (FK506) and MTX as part of a phase III prospective randomised study comparing CSP with FK506. CSP or FK506 was discontinued around day 180 post-transplant. Patients with autologous grafts received no GVHD prophylaxis.

Clinicopathological diagnosis of chronic GVHD was based on previously reported criteria. ${ }^{22}$ Patients with localised skin and/or liver dysfunction were diagnosed with limited chronic GVHD. When patients had localised skin involvement or liver dysfunction plus chronic aggressive hepatitis, eye involvement (results of Schirmer's test $\leqslant 5 \mathrm{~mm}$ ), microsalivary glands or mucosal involvement, or involvement of another target organs, we diagnosed extensive chronic GVHD. Chronic GVHD was treated with CSP (initial dose, 1 $\mathrm{mg} / \mathrm{kg} /$ day) in combination with prednisolone.

\section{STATISTICAL ANALYSIS}

The $t$ test was used to evaluate differences in the mean age in Table 1, median onset in Table 4, and MGD score in Table 5. Data are expressed as the mean (SD). Analysis of variance (ANOVA) followed by multiple comparison test (Scheffe's test) was used for statistical comparison between patients with severe dry eye, milder dry eye, and non-dry eye groups. Wilcoxon rank sum test was used to compare GVHD and non-GVHD patients. Fisher's direct method was used to evaluate differences between dry eye group and non-dry eye group in terms of sex, stem cell source, and previous acute GVHD in Table 1 and the 
Table 2 Ophthalmic complications after allogeneic SCT

\begin{tabular}{ll}
\hline Ophthalmic complications & $\%$ \\
\hline Dry eye & 50.0 \\
Meibomian gland dysfunction & 47.8 \\
Retinal haemorrhages and exudates & 25.0 \\
Acute conjunctivitis & 11.4 \\
Radiation keratopathy or Ara-C induced keratitis & 9.1 \\
Transient visual disturbance & 9.1 \\
Cataract & 6.8 \\
Allergic conjunctivitis & 6.8 \\
Iritis & 2.3 \\
\hline
\end{tabular}

incidence of dry eye and MGD with or without chronic GVHD in Table 5. A probability level of $p<0.05$ was considered statistically significant.

\section{Results}

Fifty three patients had follow up periods of at least 180 days after SCT, and were evaluable for dry eye. Their clinical profiles are analysed in Table 1. Twenty two (nine females and 13 males) out of 44 allogeneic patients $(50.0 \%)$ developed dry eye or their pre-existing dry eye worsened after SCT. During this prospective follow up, ophthalmic complications in the allogeneic group other than dry eye also were diagnosed after SCT. Those included MGD $(47.8 \%)$, retinal haemorrhages and exudates $(25.0 \%)$, acute conjunctivitis $(11.4 \%)$, radiation keratopathy or Ara-C induced keratitis $(9.1 \%)$, transient visual disturbance $(9.1 \%)$, cataract $(6.8 \%)$, allergic conjunctivitis $(6.8 \%)$, and iritis $(2.3 \%)$. Dry eye was the most frequent complication (Table 2). The mean time of dry eye onset was 171 (SD 59) days after SCT. Dry eye developed after SCT only in the allograft recipients, and not in any autograft recipients. Dry eye after SCT was observed more frequently in patients with chronic GVHD in organs other than the eyes $(70.4 \%)$ than in patients without chronic GVHD (17.7\% ; p <0.005). Before transplantation, nine out of 53 patients $(17.0 \%$; seven allograft recipients and two autograft recipi- ents) were diagnosed with dry eye (Table 1). Of these nine patients with dry eye before transplantation, four allograft recipients experienced worsening after SCT. The mean ages of allograft recipients who developed dry eye after SCT and recipients who did not were 36.0 (SD 8.0) years and 27.0 (10.7) years, respectively. The mean age of dry eye patients was significantly higher than that of non-dry eye patients $(p<0.005)$. However, there was no significant correlation between the occurrence of dry eye and recipient sex, stem cell sources (siblings $v$ unrelated donors), or previous histology of acute GVHD (Table 1).

Dry eye sensation with dry mouth was the most frequent symptom in patients with dry eye after SCT followed by foreign body sensation, ocular fatigue, discharge, and dull sensations. Recurrent conjunctivitis preceded the onset of dry eye in five patients. The results of ocular surface and tear dynamics evaluation in patients who developed dry eye before and after SCT are shown in Table 3. In this table, the worst value is presented for the posttransplant condition.

Two types of dry eye were observed (Table 3 ). In one, manifestations were severe, and ocular surface findings resembled those in Sjögren's syndrome and reflex tearing was markedly reduced. The severe type was observed in 10 cases (six females and four males). Following onset of dry eye, the conjunctival and corneal findings of severe dry eye rapidly worsened despite currently available treatment. In three cases, reflex tearing was diminished within 1 month after the onset of dry eye. In five cases the ocular surface findings preceded the tear dysfunction. The serial changes in the ocular surface and tear dynamics evaluation in 10 severe dry eye patients are shown in Figure 1. Good reflex tearing was present before SCT in 19 of 22 cases, but this began to decrease at about 3 months after SCT, and declined to $\leqslant 10 \mathrm{~mm}$ at about 6 months after SCT. In

Table 3 Clinical characteristics of patients who developed dry eye after SCT

\begin{tabular}{|c|c|c|c|c|c|c|c|c|c|c|c|c|c|c|c|c|c|c|c|c|c|}
\hline \multirow[b]{2}{*}{ No } & \multirow[b]{2}{*}{ Sex } & \multirow{2}{*}{$\begin{array}{l}\text { Age } \\
\text { (years) }\end{array}$} & \multirow[b]{2}{*}{ Diagnosis } & \multirow[b]{2}{*}{ Donor } & \multirow{2}{*}{$\begin{array}{l}\text { Onset } \\
\text { (day) }\end{array}$} & \multirow{2}{*}{$\begin{array}{l}\text { Dry } \\
\text { eye }\end{array}$} & \multirow{2}{*}{\multicolumn{2}{|c|}{$G V H D$}} & \multicolumn{2}{|c|}{$\begin{array}{l}\text { Complaints } \\
\text { (points) }\end{array}$} & \multicolumn{2}{|c|}{$F$ (points) } & \multicolumn{2}{|c|}{$\begin{array}{l}R B \\
\text { (points) }\end{array}$} & \multicolumn{2}{|c|}{$\begin{array}{l}\text { BUT } \\
\text { (seconds) }\end{array}$} & \multicolumn{2}{|c|}{$\begin{array}{l}\text { Schirmer } \\
(\mathrm{mm})\end{array}$} & \multicolumn{2}{|c|}{ NST (mm) } & \multirow{2}{*}{$\begin{array}{l}\text { MGD } \\
\text { score } \\
\text { (points) }\end{array}$} \\
\hline & & & & & & & & & pre & post & pre & post & pre & post & pre & post & pre & post & pre & post & \\
\hline 1 & $M$ & 45 & MDS & related & 83 & mild & a & c & 0 & 3 & 3 & 1 & 0 & 1 & 10 & 5 & 5 & 2 & 30 & & 0 \\
\hline 2 & M & 36 & CML & related & 180 & mild & a & c & & 22 & 0 & 3 & 0 & 2 & 10 & 5 & 19 & 14 & 26 & 23 & 0 \\
\hline 3 & $\mathrm{M}$ & 42 & ALL & related & 90 & mild & no & no & 0 & 10 & 1 & 0 & 0 & 1 & 10 & 5 & 8 & 4 & 10 & 16 & 0 \\
\hline 4 & M & 41 & AML & related & 150 & mild & a & c & 11 & 11 & 1 & 2 & 0 & 4 & 10 & 2 & 4 & 8 & 34 & 15 & 2 \\
\hline 5 & $\mathrm{~F}$ & 46 & ALL & unrelated & 90 & mild & no & no & 6 & 26 & 0 & 3 & 0 & 2 & 10 & 1 & 22 & 0 & 34 & 17 & 0 \\
\hline 6 & $\mathrm{~F}$ & 44 & MDS, AML & unrelated & 150 & mild & no & c & 2 & & 0 & 1 & 0 & 1 & 10 & 10 & 8 & 3 & 9 & & 1 \\
\hline 7 & M & 40 & AML & unrelated & 240 & mild & a & c & 3 & 7 & 0 & 4 & 0 & 4 & 10 & 5 & 11 & 8 & 15 & 33 & 1 \\
\hline 8 & $\mathrm{M}$ & 24 & AML & related & 150 & mild & $\mathrm{a}$ & c & 1 & 1 & 1 & 3 & 1 & 3 & 10 & 10 & 11 & 1 & 22 & 14 & 0 \\
\hline 9 & M & 43 & $\mathrm{MM}$ & related & 90 & mild & a & c & 1 & 4 & 0 & 3 & 0 & 3 & 10 & 4 & 16 & 6 & 33 & 17 & 1 \\
\hline 10 & $\mathrm{~F}$ & 44 & CML & related & 240 & mild & a & c & 0 & 10 & 0 & 1 & 0 & 1 & 8 & 10 & 6 & 3 & 8 & 8 & 0 \\
\hline 11 & M & 30 & ALL & unrelated & 90 & mild & no & no & 0 & 4 & 0 & 2 & 0 & 2 & 10 & 5 & 27 & 18 & 14 & 20 & 1 \\
\hline 12 & M & 37 & CML & unrelated & 240 & mild & a & c & 2 & 2 & 0 & 6 & 0 & 2 & 10 & 10 & 28 & 22 & 13 & 16 & 2 \\
\hline 13 & $\mathrm{~F}$ & 30 & AML & related & 510 & severe & a & c & 0 & 12 & 0 & 1 & 0 & 3 & 10 & 5 & 5 & 3 & 12 & 5 & 1 \\
\hline 14 & $\mathrm{~F}$ & 21 & AML & unrelated & 180 & severe & a & c & 4 & 30 & 2 & 7 & 0 & 7 & 10 & 2 & 13 & 4 & 26 & 1 & 2 \\
\hline 15 & $\mathrm{~F}$ & 25 & ALL & related & 109 & severe & a & c & 2 & 8 & 0 & 3 & 0 & 4 & 10 & 5 & 4 & 6 & 3 & 5 & 1 \\
\hline 16 & M & 33 & AML & unrelated & 180 & severe & no & c & 0 & 8 & 1 & 5 & 0 & 5 & 10 & 8 & 35 & 6 & 34 & 6 & 2 \\
\hline 17 & M & 29 & CML & related & 168 & severe & no & c & 0 & 9 & 0 & 6 & 0 & 7 & 10 & 2 & 32 & 4 & 35 & 4 & 2 \\
\hline 18 & $\mathrm{~F}$ & 22 & ALL & related & 120 & severe & a & c & 0 & 38 & 0 & 6 & 0 & 5 & 10 & 3 & 12 & 2 & 27 & 3 & 2 \\
\hline 19 & $\mathrm{~F}$ & 43 & MDS & unrelated & 150 & severe & a & c & 9 & 16 & 1 & 4 & 3 & 3 & 8 & 2 & 1 & 0 & 4 & 1 & 2 \\
\hline 20 & M & 35 & $\mathrm{MM}$ & related & 210 & severe & a & c & 0 & 18 & 0 & 5 & 0 & 3 & 10 & 8 & 12 & 3 & 29 & 7 & 2 \\
\hline 21 & M & 43 & MDS & unrelated & 135 & severe & a & c & 0 & 9 & 0 & 2 & 0 & 1 & 10 & 2 & 9 & 3 & 15 & 9 & 2 \\
\hline 22 & $\mathrm{~F}$ & 35 & CML & unrelated & 200 & severe & a & c & 15 & 32 & 2 & 6 & 2 & 5 & 9 & 2 & 4 & 0 & 3 & 0 & 2 \\
\hline
\end{tabular}

SCT $=$ stem cell transplantaion; GVHD $=$ GVHD in organs other than the eyes; $\mathrm{F}=$ fluorescein score $; \mathrm{RB}=$ rose bengal score; $\mathrm{BUT}=$ tear break up time; $\mathrm{NST}=$ Schirmer test with nasal stimulation; MGD = meibomian gland dysfunction; pre $=$ pre-SCT ; post $=$ post-SCT; $\mathrm{a}=\mathrm{acute} ; \mathrm{c}=\mathrm{chronic} ; \mathrm{MDS}=$ myelodysplastic syndrome; $\mathrm{CML}=$ chronic myeloid leukaemia $; \mathrm{ALL}=$ acute lymphoblastic leukaemia; $\mathrm{AML}=$ acute myeloid leukaemia; $\mathrm{MM}=$ multiple myeloma. 


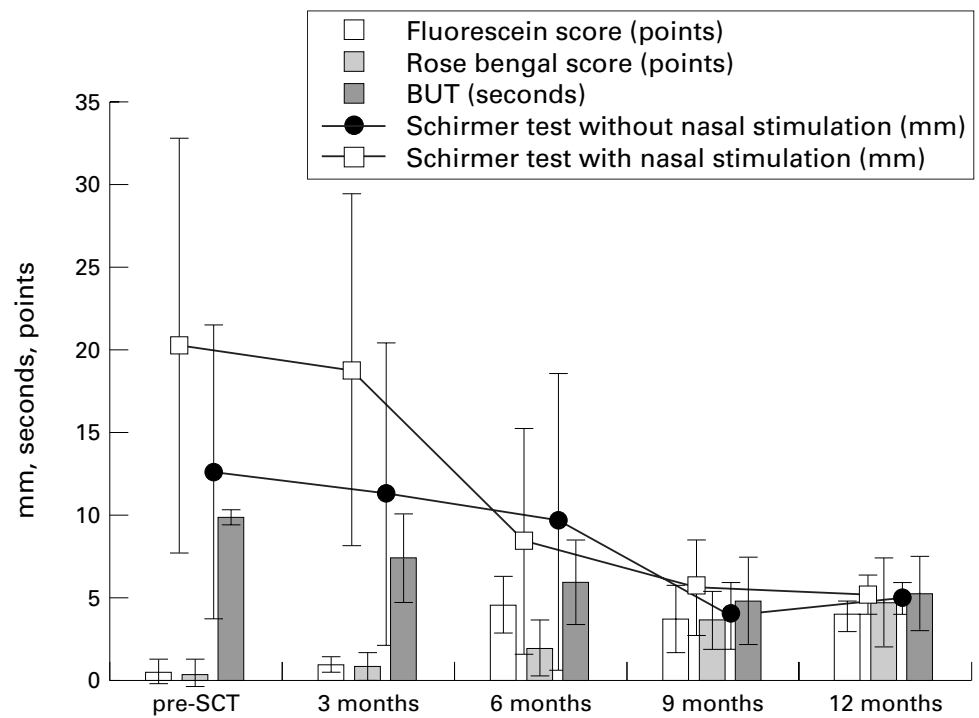

Figure 1 The serial changes in the ocular surface and tear dynamics evaluation in 10 severe dry eye patients are shown. Good reflex tearing was present before stem cell transplantation (SCT), but this began to decrease at about 3 months after SCT and declined to $\leqslant 10 \mathrm{~mm}$ at about 6 months after SCT.

contrast, the mean value of Schirmer tests without nasal stimulation declined to $\leqslant 5 \mathrm{~mm}$ at about 9 months after SCT. Keratoconjunctivitis occurred or worsened during the period of 3-6 months after SCT (Fig 1). In mild type, the degree of dry eye was mild without reduced reflex tearing. This type was observed in 12 cases (three females and nine males). Their symptoms and ocular surface findings of dry eye were mild and, were controlled by using commercially available artificial tears (Table 3).

Severe dry eye developed more frequently in patients with chronic GVHD in organs other than the eyes $(\mathrm{p}<0.001)$, and median time from onset of severe dry eye was later than mild dry eye (196 (115) days; range 109-510 days $v$ 149 (63) days; range 83-240 days) after SCT, respectively, but the difference was not significant. The diagnosis of severe dry eye did not significantly correlate with recipient sex, stem cell source, or previous history of acute GVHD. Severity of dry eye correlated with the presence of chronic GVHD and MGD $(\mathrm{p}<0.001 ;$ Table 4$)$.

MGD was recognised more frequently in patients with chronic GVHD (63.0\%) than in

Table 4 Clinical characteristics of two types of dry eye after allogeneic SCT

\begin{tabular}{|c|c|c|c|c|}
\hline & $\begin{array}{l}\text { Severe dry eye } \\
(n=10)\end{array}$ & $\begin{array}{l}\text { Mild dry eye } \\
(n=12)\end{array}$ & $\begin{array}{l}\text { Non-dry eye } \\
(n=22)\end{array}$ & \\
\hline Mean age (years) (SD) & $31.8(7.9)$ & $39.5(6.4)$ & $27.7(10.7)$ & \\
\hline \multicolumn{5}{|l|}{ Sex } \\
\hline$M$ & $4(40.0 \%)$ & $9(75.0 \%)$ & $15(68.2 \%)$ & NS \\
\hline $\mathrm{F}$ & $6(60.0 \%)$ & $3(25.0 \%)$ & $7(31.8 \%)$ & \\
\hline Median onset (days) & $196(115)$ & $149(63)$ & & NS \\
\hline \multicolumn{5}{|l|}{ Stem cell source } \\
\hline related donor & $5(50 \%)$ & $7(58.3 \%)$ & 17 & NS \\
\hline unrelated donor & $5(50 \%)$ & $5(41.7 \%)$ & 5 & \\
\hline Acute GVHD (+) & $8(80.0 \%)$ & $9(75.0 \%)$ & $11(50 \%)$ & NS \\
\hline Acute GVHD (-) & $2(20.0 \%)$ & $3(25.0 \%)$ & $11(50 \%)$ & \\
\hline Chronic GVHD (+) & $10(100 \%)$ & $9(75.0 \%)$ & $8(36.4 \%)$ & $\mathrm{p}<0.001$ \\
\hline Chronic GVHD (-) & $0(0 \%)$ & $3(25.0 \%)$ & $14(63.6 \%)$ & \\
\hline $\operatorname{MGD}(+)$ & $10(100 \%)^{\star \star \star}$ & $6(50.0 \%)^{\star}$ & $5(22.7 \%)^{\star \star}$ & $\mathrm{p}<0.05^{\star}, \mathrm{p}<0.001^{\star \star}$ \\
\hline MGD (-) & $0(0 \%)$ & $6(50.0 \%)$ & $17(77.3 \%)$ & \\
\hline MGD score & $1.8(0.4)^{\star \star \star}$ & $0.7(0.87)^{\star}$ & $0.4(0.7)^{\star \star}$ & $\mathrm{p}<0.005^{\star}, \mathrm{p}<0.001^{\star \star}$ \\
\hline
\end{tabular}

GVHD $=$ graft versus host disease in organs other than the eyes; MGD = meibomian gland dysfunction; SCT $=$ stem cell transplantation.
Table 5 Correlations between dry eye or MGD and chronic GVHD

\begin{tabular}{llll}
\hline & $\begin{array}{l}\text { Chronic } \\
\text { GVHD (+) } \\
n=27\end{array}$ & $\begin{array}{l}\text { Chronic } \\
\text { GVHD (-) } \\
n=17\end{array}$ & p Value \\
\hline $\begin{array}{l}\text { Dry eye (+) } \\
\text { Dry eye (-) }\end{array}$ & $\begin{array}{l}19(70.4 \%) \\
8(29.6 \%)\end{array}$ & $\begin{array}{l}3(17.7 \%) \\
14(82.3 \%)\end{array}$ & $<0.005$ \\
$\begin{array}{l}\text { Severity of dry eye } \\
\text { Severe }\end{array}$ & $10(37.4 \%)$ & $0(0.0 \%)$ & $<0.001$ \\
$\quad \begin{array}{l}\text { Mild } \\
\text { Dry eye (-) }\end{array}$ & $\begin{array}{l}9(33.3 \%) \\
8(29.6 \%)\end{array}$ & $\begin{array}{l}3(17.7 \%) \\
14(82.3 \%)\end{array}$ & \\
MGD (+) & $17(63.0 \%)$ & $4(23.5 \%)$ & $<0.05$ \\
MGD (-) & $10(37.0 \%)$ & $13(76.5 \%)$ & $<0.005$ \\
MGD score (SD) & $1.1(0.9)$ & $0.3(0.6)$ & $<0.005$ \\
\hline
\end{tabular}

GVHD $(+)=$ graft versus host disease in organs other than the eyes $(+) ; \mathrm{MGD}=$ meibomian gland dysfunction.

patients without chronic GVHD (23.5\%; $\mathrm{p}<0.05$ ), and MGD score was also higher in patients with chronic GVHD than without chronic GVHD ( $\mathrm{p}<0.005$; Table 5).

\section{Discussion}

We found that half of allogeneic SCT patients developed dry eye or their pre-existing dry eye worsened after transplant. This incidence is much higher than those reported in previous prospective 5 $^{610}$ and retrospective 7 $^{911}$ studies. In previous reports, dry eye after SCT was diagnosed as evaporative and/or tear deficient type using classic or simple diagnostic tests. We used the diagnostic criteria of dry eye including both evaporative and tear deficient type, ${ }^{19}{ }_{20}$ extensively applied various modern diagnostic tests including Schirmer test with nasal stimulation and evaluation of MGD. ${ }^{15} 18$ The difference in both diagnostic criteria and tools may explain the higher incidence of dry eye in this prospective study. Nine patients $(17.0 \%$; median age 33 years) had dry eye before SCT in this prospective study. The incidence was similar to that previously reported using the same diagnostic criteria as our study. ${ }^{23}$

In this study, none of the patients who received autografts developed dry eye or had worsening of existing dry eye; this ocular complication was clearly limited to allograft recipients. The median time from transplantation to diagnosis of dry eye was 171 (59) days. Administration of immunosuppressant drugs for GVHD prophylaxis is usually completed by about 180 days after SCT, and chronic GVHD frequently develops during tapering or shortly after discontinuation of immunosuppression. One case of dry eye was diagnosed 510 days after SCT, soon after cessation of long term systemic immunosuppressive therapy for chronic GVHD in other organs. The median time from SCT to diagnosis of dry eye tended to coincide with the time when chronic GVHD was frequently observed. These facts suggests that an alloimmune process contributes actively to the pathogenesis of dry eye after SCT.

The distinction that we observed between severe and mild dry eye after allogeneic SCT has not been reported previously. The severe form was observed more frequently with chronic GVHD, suggesting that a posttransplant alloimmune reaction plays a significant part in the development of severe dry eye after SCT. Severe dry eye was not associated with corneal ulcer or conjunctival scarring, ${ }^{24} 25$ 
possibly because of the early detection of dry eye in this prospective study with close follow up. It remains unclear whether mild dry eye is a disease with a different aetiology or is an early stage of severe dry eye. We need more patients with a longer follow up to definitively answer this question.

Meibomian gland function was severely damaged in patients with severe dry eye and chronic GVHD. Our results also suggested that MGD may allow us to diagnose severe dry eye with GVHD early in the course of the disease. Meibomian glands are specialised sebaceous glands. It is thought that the bulge of hair follicles located below the opening of the sebaceous duct is destroyed by donor $\mathrm{T}$ cells in chronic GVHD. ${ }^{26}{ }^{27}$ This seems to result in MGD. Jester et al showed that hyperkeratinisation of the meibomian duct epithelium leading to plugging and dilatation of the duct underlies the development of MGD in a rabbit model. ${ }^{28}$ We also observed that ocular surface changes preceded the deterioration of tear function in five cases of severe dry eye. Keratinisation may be an another possible mechanism for MGD with GVHD, although the pathogenesis remains controversial.

Although chronic GVHD is the most important cause of dry eye after SCT, total body irradiation, ocular toxicity of chemotherapy, infections, and immunosuppressive therapy may also contribute to dry eye after SCT. ${ }^{135} 1029$

Livesey et al reported the development of keratoconjunctivitis sicca in $81.8 \%$ of patients with chronic GVHD and $33.3 \%$ of patients after autologous SCT. ${ }^{7}$ They suggested that orbital irradiation even at the dose used for conditioning contributes to development of KCS. Therefore, dry eye following SCT is not uniquely associated with allogeneic SCT and chronic GVHD. However, our observations were quite different. Two of the seven autograft recipients who had dry eye before SCT did not experience worsening after TBI. One of our patients was treated with local radiotherapy to an orbital tumour 1 year before SCT, but this case was found to have normal tear dynamics and ocular surface just before SCT. Dry eye did not occur until after allogeneic SCT in this case. Parsons et al observed that severe dry eye developed during or shortly after TBI treatment in most cases. ${ }^{30}$ We observed keratopathy shortly after SCT and TBI, but this resolved by 3 months after SCT. Our findings suggest that low dose fractionated TBI has little influence on the late onset dry eye (observed $>100$ days ) after SCT.

MTX has mucosal toxicity and affects the meibomian glands. ${ }^{31}$ CSP also is known to affect mucosal membranes, and can induce gingival and conjunctival hyperplasia. ${ }^{10} 32$ Ara-C may influence the ocular surface resulting in keratoconjunctivitis. ${ }^{334}$ Typically, symptoms are first noticed 4-7 days after commencement of therapy. We observed typical Ara-C induced keratitis in seven cases, but symptoms and signs of keratoconjunctivitis had resolved in all cases by 3 months after SCT. In addition, we used Ara-C even in the autologous and allogeneic patients who did not develop dry eye or MGD after SCT. Also, having patients observed for up to 30 months after SCT and finding normal meibomian gland function without dry eye, we doubt that MTX, CSP, or Ara-C significantly affects meibomian gland function or predisposes to dry eye after SCT.

The Schirmer test has been used as a gold standard for diagnosis of extensive chronic GVHD involving the eye, although its specificity and sensitivity for diagnosis of dry eye has been questioned. When dry eye for extensive chronic GVHD was diagnosed according to the criteria used in this study, ${ }^{19}{ }^{20}$ the false positive diagnosis rate for dry eye due to chronic GVHD by Schirmer test without nasal stimulation was $19.4 \%$ (six of 31 cases) with false negative representing $36.4 \%$ (eight of 22 cases). Reduction of the mean value of the Schirmer tests with nasal stimulation correlates well with chronic GVHD in organs other than the eyes, but no correlation between the mean results of the Schirmer test without nasal stimulation and chronic GVHD was found. We therefore would emphasise the importance of the Schirmer test with nasal stimulation including ocular surface evaluation ${ }^{16-20}$ to better assess the diagnosis of dry eye associated with chronic GVHD. Dry eye is a major complication after SCT and should be recognised and dealt with. Further study for the diagnosis and treatment should be continued, with a focus on reduced reflex tearing and MGD.

We thank Drs S Suzuki, J Shimazaki, and S Shimmura for their useful suggestions. We thank the orthoptists and photographer Mr Seki, Keio University for their assistance.

1 Ferrara JL, Deeg HJ. Graft-versus-host disease. $N$ Engl $\mathcal{F}$ Med 1991;324:667-74.

2 Thomas ED, Storb R. The development of the scientific foundation of hematopoietic cell transplantation based on animal and human studies. In: Thomas ED, Blume KG, Forman SJ, eds. 2nd ed. Haematopoietic cell transplantation.

3 Atkinson K Review: Chronic graft-versus-host disease. Bone Marrow Transplant 1990;5:69-82.

4 Sullivan KM. Graft-versus-host disease. In: Thomas ED, Sullivan KM. Graft-versus-host disease. In: Thomas ED,
Blume KG, Forman SJ, eds. 2nd ed. Haematopoietic cell transplantation. Oxford: Blackwell, 1998:515-36.

5 Hirst LW, Jabs DA, Tutschka PJ, et al. The eye in bone marrow transplantation. I. Clinical study. Arch Ophthalmol 1983;101:580-4

6 Calissendorff B, Azazi ME, Lönnqvist B. Dry eye syndrome in long-term follow-up of bone marrow transplanted patients. Bone Marrow Transplant 1989;4:675-8.

7 Livesey SJ, Holmes JA, Whittaker JA. Ocular complications of bone marrow transplantation. Eye 1989;3;271-6.

8 Tanabe S, Hirano J, Murakami M. Manifestations and treatment of ocular graft-versus-host disease in allogeneic bone marrow transplantation. Fpn $\mathcal{F}$ Clin Ophthalmol 1992; 46:1749-52.

9 Tichelli A, Duell T, Weiss M, et al. Late-onset keratoconjunctivitis sicca syndrome after bone marrow transplantation: incidence and risk factors. Bone Marrow Transplant. 1996;17:1105-11.

10 Mencucci R, Ferrini CR, Bosi A, et al. Ophthalmological aspects in allogeneic bone marrow transplantation: Sjögren-like syndrome in graft-versus-host disease. Eur $\mathcal{F}$ Ophthalmol 1997;7:13-8.

11 Yoshino M, Yamada M, Ogawa Y, et al. Dry eye associated with bone marrow transplantation. Folia Ophthalmol fpn 1997;48:453-5.

2 Toda I, Shinozaki N, Tsubota K. Hydroxypropyl methylcellulose for the treatment of severe dry eye associated with Sjögren's syndrome. Cornea 1996;15:120-8.

13 Toda I, Tsubota K. Practical double vital staining for ocular surface evaluation. Cornea 1993;12:366-8.

14 Tsubota K, Toda I, Yagi Y, et al. Three different types of dry eye syndrome. Cornea 1994;13:202-9.

$15 \mathrm{Xu} \mathrm{K}$, Yagi Y, Toda I, et al. Tear function index: a new measure of dry eye. Arch Ophthalmol 1995;113:84-8.

16 Tsubota K. The importance of Schirmer test with nasal stimulation. Am f Ophthalmol 1991;111:106-8.

17 Tsubota K, Xu K, Fujihara T, et al. Decreased reflex tearing is associated with lymphocyte infiltration in lacrimal glands. $\mathcal{F}$ Rheumatol 1996;23:313-20. 
18 Shimazaki J, Sakata M, Tsubota K. Ocular surface changes and discomfort in patients with meibomian gland dysfuncand discomfort in patients with meibomia
tion. Arch Ophthalmol 1995;113:1266-70.

19 Shimazaki J. Definition and criteria of dry eye. Ganka Shimazaki J. Definition
(Japan) 1995;37:765-70.

20 Lemp MA. Report of the National Eye Institute/Industry Workshop on Clinical Trials in Dry Eyes. CLAO $\mathcal{F}$ 1995;21:221-32.

21 Shimazaki J, Goto E, Ono M, et al. Meibomian gland dysfunction in patients with Sjögren's syndrome. Ophthalmology 1998;105:1485-8.

22 Schulman HM, Sullivan KM, Weiden PL, et al. Chronic graft-versus-host syndrome in man. A long-term clinicopathologic study of 20 Seattle patients. Am ₹ Med 1980;69. 204-17.

23 Hikichi T, Yoshida A, Fukui Y, et al. Prevalence of dry eye in Japanese eye centers. Graefes Arch Clin Exp Ophthalmol 1995;233:555-8.

24 Jack MK, Jack GM, Sale GE, et al. Ocular manifestations of graft-v-host disease. Arch Ophthalmol 1983;101:1080-4.

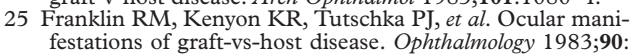
festations.

26 Sale GE, Beauchamp MD, Akiyama M. Parafollicular bulges, but not hair bulb keratinocytes, are attacked in graft-versus-host disease of human skin. Bone Marrow Transplant 1994;14:411-13.
27 Akiyama M, Dale BA, Sun TT, et al. Characterization of hair follicle bulge in human fetal skin:The human fetal bulge is a pool of undifferentiated keratinocytes. F Invest Dermatol 1995;105:844-50.

28 Jester JV, Nicolaides N, Kiss-Palvolgyi I, et al. Meibomian gland dysfunction II. The role of keratinization in a rabbit model of MGD. Invest Ophthalmol Vis Sci 1989;30:936-45.

29 Bray LC, Carey PJ, Proctor SJ, et al. Ocular complications of bone marrow transplantation. Br f Ophthalmol 1991;75: 611-14

30 Parsons JT, Fitzgerald CR, Hood CI, et al. The effects of irradiation on the eye and optic nerve. Int $\mathcal{F}$ Radiat Oncol Biol Phys 1983;9:609-22.

31 Fraunfelder FT, Meyer SM. Ocular toxicity of antineoplastic agents. Ophthalmology 1983;90:1-3.

32 Nussenblatt RB, Palenstine AG. Therapeutic review. Cyclosporine: immunology, pharmacology and therapeutic uses. Surv Ophthalmol 1986;31:159-69.

33 Barletta JP, Fanous MM, Margo CE. Corneal and conjunctival toxicity with low-dose cytosine arabinoside. Am $\mathcal{F}$ Ophthalmol 1992;113:587-8.

34 Lass JH, Lazarus,HM, Reed MD, et al. Topical corticosteroid therapy for corneal toxicity from systemically administered cytarabine. Am f Ophthalmol 1982;94:61721 